\title{
User-extensible sequences in Common Lisp
}

\author{
Christophe Rhodes \\ Goldsmiths, University of London \\ New Cross Road, London, SE14 6NW \\ c.rhodes@gold.ac.uk
}

\begin{abstract}
Common Lisp is often touted as the programmable programming language, yet it sometimes places large barriers in the way, with the best of intentions. One of those barriers is a limit to the extensibility by the user of certain core language constructs, such as the ability to define subclasses of built in classes usable with standard functions: even where this could be achievable with minimal penalties. We introduce the notion of user-extensible sequences, describing a protocol which implementations of such classes should follow. We show examples of their use, and discuss the issues observed in providing support for this protocol in a Common Lisp, including ensuring that there is no performance impact from its inclusion.
\end{abstract}

\section{INTRODUCTION}

Common Lisp is recognized as being an extremely flexible language: one in which linguistic experimentation can take place, one where the method of solving a problem in a domain is first to write an interpreter or a compiler for a language to express concepts in the domain of interest, and then to use that domain-specific language to solve the problem. To some extent, then, it might be surprising to find some of the ways in which extension of Common Lisp (by the implementor or user) is limited. This is at least partially explained by the aims of the standardizers, which included "stricter standardization for portability" (Pitman and Chapman, 1994, section 1.1.2): codification of existing practice was a large part of the X3J13 committee's work, and it is difficult to ensure portability with a highly-extensible language core.

However, the standardization process was not intended to close the door to language development: merely to provide a stable platform which could be agreed on. For example, the CLOS system for object orientation was standardized without very much scope for extensibility; however, it was intentionally (Steele, 1990, chapter 28) upwardly compatible with something close to the Metaobject Protocol described in Kiczales et al. (1991), often (though not always) supported by contemporary Lisp implementations.

Meanwhile, other languages and language environments have not stood still; many of Lisp's once-unique features are now to be found in other languages (Norvig, 2002), though some are still not ${ }^{1}$ additionally, sometimes these languages have features not found in any available Lisp implementation. In some cases, such as Aspect-Oriented Programming (Kiczales et al. 1997) these features can be straightforwardly implemented by any interested user - this itself is one of Lisp's unique features not often found elsewhere - but some features need implementation support for them to be used to maximum effect: addition of features by the Lisp programmer can suffice for certain needs, but they are not pervasive in the way that one would want; such extensions, even upwardly-compatible extensions, need to be explicitly used by third-party code.

One such extension is the ability for the user to define new sequence types. For the purposes of this paper, a sequence is a finite ordered collection; more concretely, a sequence has a number of elements, addressable by an integer between 0 (inclusive) and the sequence's length (exclusive). The motivation for allowing the definition of new sequence types is primarily to eliminate the need for new names for essentially the same concept: examples of such from real-world code include climacs-buffer:size and flexichain:nbelements (Strandh et al. 2004) as analogues for length on user-defined data structures, and tabcode-syntax:bufferposition-if (Rhodes et al., 2005) for position-if these multiple names are a barrier to clear expression and understanding of Common Lisp code which defines objects which are conceptually sequences. Additionally, the implementation of sequence functionality is tedious and error-pron $\AA^{2}$ and it is more convenient to require of the datastructure implementor only a few simple method definitions to achieve all the standard sequence functionality.

We describe in this paper a proposal for implementations to allow users to define new sequence classes which interoperate seamlessly with the standard Common Lisp sequence functions. The basic mode of operation of our design is as

\footnotetext{
${ }^{1}$ and it has been argued that their inclusion would convert their host language into a Lisp of some form.

${ }^{2}$ The author, in developing this extension, wrote the implementation first and test cases second: the test cases revealed five implementation errors.
} 


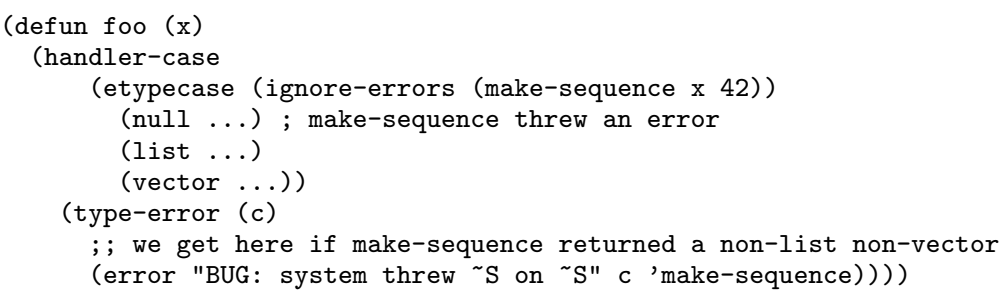

Figure 1: How to detect formally nonconforming implementations of make-sequence. If calling foo with any argument causes the type-error handler to be called, the Common Lisp implementation is, strictly speaking, nonconforming.

follows: implementors of sequence classes write methods on generic functions named by symbols in the sequence package; users of sequence classes call the standard functions named by symbols in the common-lisp package: it is intended that sufficiently generic existing code may be run unmodified using extended sequences. The implementor of this proposal is responsible for arranging that the calls to the standard functions invoke applicable methods on the appropriate functions in the sequence package. Further, users wishing to construct new functions involving iteration over sequences should call the iteration operators included in this proposal, so that their new functions will work on user-defined sequence classes.

The rest of this paper is organized as follows: we introduce the design considerations of this proposal in section 1.1 then, after discussing compatibility issues and related work in sections 1.2 and 1.3 we give an introduction for the prospective user of extensible sequences in section 2 , and some examples in section 3 , and some details of our implementation are given in section 4 As a snapshot of our work in progress, a more formal specification of the protocols is presented in appendix $\mathrm{A}$ intended in the first instance to stimulate discussion rather than to be the definitive specification.

\subsection{Design considerations}

Our design for this extension as a whole was influenced by a number of desiderata:

- We wish to minimize incompatibility with the existing Common Lisp standard, introducing such incompatibility only where strictly necessary. There are only a few corner cases where the existing standard document needs to be clarified or reinterpreted; see section 1.2 for discussion of this point.

- We wish to make defining new sequence classes simple and convenient for users, so that simply defining a subclass of sequence and a few methods specialized on that class suffices for the entire sequence functionality to be available, and also to provide enough hooks for sequence class implementors to be able to customize the behaviour for their own needs.

- We wish not to overly constrain implementations of this protocol, allowing implementors to make tradeoffs to reflect the needs of their users. In particular, we wish to allow both a simple implementation (where the Common Lisp implementation supports this protocol natively for all sequence types) and an implementation where existing code suffers no run-time performance penalty; additionally, we do not wish to render a portable implementation impossible, though we believe that such an implementation would necessarily have visible seams.

While it is possible to recommend ways of accessing and iterating over both Common Lisp sequences and other objects in userspac $\xi^{3}$ there is no way of having those other objects (conceptually sequences though not of type sequence) seamlessly interoperate with the standard Common Lisp sequence functionality, or with third-party code which does not follow such a recommendation.

The desire not to constrain implementations of this proposal leads to the creation of a new package name to name the operators providing the extensible functionality, allowing dispatch from the standard function to the extensible one through trampolining or defadvice-like mechanisms. However, an implementation of Common Lisp wishing to support this proposal natively may choose to implement some of its CL symbols as direct imports from the new sequence package.

In what follows, there will be reference to new operators without explicit package prefixes: in such cases, the symbols' package should be assumed to be the sequence package. A number of new operators have the same name as standardized operators of Common Lisp; except in appendix A the operator without an explicit prefix should be taken to mean the Common Lisp operator, while the new operators will have an explicit sequence: prefix.

\subsection{Compatibility}

The types $[s i c]$ vector and the type list are disjoint subtypes of type sequence, but are not necessarily an exhaustive partition of sequence.

Pitman and Chapman (1994, System Class sequence)

Although the quote above might suggest that there would be no problem from the point of view of formal standard con-

\footnotetext{
${ }^{3}$ By 'userspace' in this paper, we mean the realm of conforming or de-facto acceptable code written by users of an implementation, as opposed to code written by implementors themselves to provide an implementation with extensions.
} 


\begin{tabular}{|c|c|}
\hline $\begin{array}{l}\text { sequence: length } \\
\text { sequence: adjust-sequence }\end{array}$ & $\begin{array}{l}\text { sequence:elt } \\
\text { sequence:make-sequence-like }\end{array} \quad$ (setf sequence:elt) \\
\hline
\end{tabular}

Table 1: The protocol functions which must be implemented for a sequence class. Three correspond directly to primitive sequence operations in Common Lisp; adjust-sequence and make-sequence-like provide support for components of higher-level destructive and consing operations respectively (see table 2 ).

formance for an implementation to offer non-standard types of sequence the outline code in figure 1, as a result of Issue CONCATENATE-SEQUENCE (Pitman, 1991), is defined by ANSI CL never to get to the type-error branch of the handlercase, which was probably not intended by the standardizers. For a fuller discussion of this issue, and a suggestion for a resolution, see Rhodes (2006).

An additional issue with formal compliance is that ANSI CL specifies for the length function and the elt accessor that their sequence argument should be a proper sequence, which is defined in the normative glossary as a sequence which is not an improper list; that is, a vector or a proper list; again, this definition was probably not intended to prohibit nonstandardized sequences, even though, interpreted strictly, the second half of it implies that no non-standard sequence is a proper sequence.

We must also consider the issue of cultural compatibility with the body of Common Lisp code available in the wild. Perhaps because of the historical lack of generic sequences offered by Common Lisp implementations, there seems to be little in the way of packages written to exploit the abstract sequence data type; instead, packages choose a concrete sequence type and implement their functionality atop that, manipulating a particular kind of sequence rather than sequences in genera $4^{4}$ However, it is likely that if userextensible sequences become available, code will be modified or written afresh to take advantage of it.

It would be possible to implement this proposal (or something like it) entirely portably (in 'userspace'), by defining a new package shadowing many of the standard Common Lisp functions and macros, and implementing the generic functionality on the standard types by trampolining to the standard functions. However, such an implementation strategy has drawbacks:

- Implementation-specific compiler optimizations (such as compiler macros) for the shadowed functions would essentially be lost. As an example, if an implementation has a specialized implementation of map-into for arguments known at compile-time to be certain types of vector, that optimization will go unused in a putative userspace implementation in function calls to sequence-cl:map-into.

- The userspace implementation of extensible sequences would not interoperate with third-party Common Lisp code: programs already written with generic (not nec-

\footnotetext{
${ }^{4}$ There are exceptions: for instance, the split-sequence library, designed by readers of news: comp.lang.lisp in 2001, works without modification on generic sequences of the form described in this paper.
}

essarily user-extensible) sequences in mind, using the common-lisp package, will not be able to interoperate cleanly with sequences defined using this portable implementation.

- Innocuous-looking uses of explicit package prefixes (for example, cl: sequence) would have surprising and potentially difficult-to-debug effects. Other maintenance headaches include how to support both native and portable implementations in library code.

A userspace implentation might be better than nothing, for a transition period, but support from the Lisp implementation is required for seamless operation.

That said, there is an issue regarding portability of libraries using extensible sequences: until it is ubiquitously implemented, use of this facility in bodies of code render those bodies of code unportable in practice. Whether this unportability itself becomes a problem in practice is largely a matter for the user community to decide.

\subsection{Related Work}

Much inspiration for this proposal was drawn from the Dylan (Shalit, 1996) iterator protocol, which provides for iteration over collections with the open generic function forwarditeration-protocol. Where our design is similar to this protocol, it is largely for the same reasons: we do not wish to impose unnecessary run-time overhead (in space or speed) for those uses which need high performance. However, in this proposal, we aim to provide a little more of a layer of convenience for the user who does not need to minimize overhead.

Many other languages provide some form of iteration or collection protocol. Python, for instance, allows the implementor of a collection to define a method on _-_iter_-( ) to return an iterator object, which itself must have methods on _-_iter_-_() and next(). Apparently for reasons of efficiency, the Python iterator protocol (Yee and van Rossum. 2001) provides no explicit means for checking for termination of an iteration, instead requiring the iterator to signal an exception when next () is called on an iterator representing a terminated iteration.

The Scheme language (Kelsey et al., 1998) definition has little built-in support for user-extensible or even generic sequences. Its community has made one attempt (Miller. 2004) at defining an interface and conventions for collections; however, this SRFI has apparently not seen many Scheme implementations decide to support it natively; as of two years after its finalization, the SRFI status page reports no implementation as supporting it. 


\begin{tabular}{|c|c|}
\hline Common Lisp Function & Extensible Generic Function \\
\hline copy-seq, subseq & sequence: copy-seq, sequence: subseq \\
\hline reduce $\mathrm{I} i \perp$ & sequence: reduce, sequence: fill \\
\hline reverse, nreverse & sequence:reverse, sequence: nreverse \\
\hline sort, stable-sort & sequence: sort, sequence: stable-sort \\
\hline count find position & sequence: count, sequence:find, sequence:position \\
\hline count-1t, count-1t-not & sequence: count-if, sequence: count-if-not \\
\hline Iınd-ıt, fınd-ıt-not & sequence: find-if, sequence: find-if-not \\
\hline position-1t, position-if-not & sequence:position-if, sequence:position-if-not \\
\hline search mismatch replace & sequence: search, sequence:mismatch, sequence:replace \\
\hline substıtute, nsubstıtute & sequence: substitute, sequence: nsubstitute \\
\hline substıtute-1t, substıtute-if-not & sequence: substitute-if, sequence: substitute-if-not \\
\hline nsubstıtute-ıf, nsubstıtute-ıt-not & sequence: nsubstitute-if, sequence: nsubstitute-if-not \\
\hline remove delete & sequence:remove, sequence: delete \\
\hline remove-it remove-if-not & sequence:remove-if, sequence:remove-if-not \\
\hline delete-1t, delete-1t-not & sequence: delete-if, sequence: delete-if-not \\
\hline remove-duplicates, delete-duplicates & sequence:remove-duplicates, sequence:delete-duplicates \\
\hline
\end{tabular}

Table 2: Common Lisp functions, and the corresponding generic functions specified to be extensible in this protocol. Calling the Common Lisp function with an extended sequence as an argument will cause applicable methods on the extensible generic function to be invoked; whether this is achieved through defadvice, trampolining or function identity is left unspecified.

The Factor language's sequence interface is conceptually very similar to that described in the following sections for Common Lisp, including the distinction between sequence protocol (defining the fundamental operations that must be implemented for sequence objects) and 'utility words', analogous to functions performing computations over sequences (Pestov, 2006, Sequences), which will work on any object implementing the sequence protocol.

In the Common Lisp world itself, an early (pre-CLOS) attempt to provide generic sequence functionality was presented in Haible (1988) for the GNU CLISP implementation; however, that proposal was never formally exported or documented (Haible, 2006); the author's primary concern with this attempt was in supporting the necessary operations efficiently. Some of these issues of efficiency remain in this proposal, though we believe that they might be resolved in a Lisp implementation supporting sealing and inlining of methods.

\section{USER-DEFINED SEQUENCES}

In this section, we must draw the distinction between the implementor of a sequence class, and the implementation of Common Lisp which supports this user-extensible sequence facility. Most of the time, we will use 'user' to mean the implementor of a sequence class, and 'implementor' to mean implementor of a Common Lisp implementation, and we hope that it will be clear from context when this does not apply.

The names of the various operators have been chosen to maximize both backward- and forward-compatibility (with Common Lisp as standardized, and with potential related extensions to Common Lisp such as a collections protocol); we specify operators corresponding to standardized functions such as find to be named like sequence: $f$ ind, so that an implementation of Common Lisp 'natively' implementing this proposal can simply import the relevant symbols from the sequence packag $5^{5}$ Thus, we specify sequence:adjust-

\footnotetext{
${ }^{5}$ According to some interpretations of the standard, the im-
}

sequence rather than (setf sequence:length), so that an implementation can import sequence:length into the standard common-lisp package without inadvertantly causing there to be a setf function for length, in contravention of the constraints on implementations (Pitman and Chapman 1994, Section 11.1.2.1.1).

We also aim to be not incompatible with similar extensions to Common Lisp, such as a protocol for accessing and iterating over general collections; in this proposal we are dealing with user-defined sequence classes because Common Lisp as standardized has a large library of functions acting on generic sequences, while it has no functions acting on generic collections - and so a 'userspace' implementation of a collections protocol would not pose the interoperability problems discussed in section 1.2

\subsection{Sequence Datatypes}

Under this proposal, the user may define a direct subclass of sequence using defclass, specifying sequence as one of the superclasses (but not the only one: for code portable between implementations of this proposal, standard-object must be in the superclasses list too). The resulting class is subtypep sequence and instances of the class are typep sequence

The fundamental operations defined in Common Lisp relating to sequences are length, elt, and (setf elt); in order to support these, the user of the extensible sequence facility (the implementor of the sequence class) should define methods on sequence:length, sequence: elt and (setf sequence:elt).

plementation may not simply make sequence a nickname for the common-lisp package, as the list of package nicknames is standardized as cl only.

${ }^{6}$ Reviewers of this paper have raised concerns about the use of sequence as a direct superclass, suggesting instead a protocol class, maybe sequence-object, as the userspecializeable class. The author wishes to acknowledge reviewer concerns in this matter, while continuing to present the protocol as currently implemented. 
The set of functions making up the sequence protocol of this proposal (see table 1) consists of the analogues to the three Common Lisp functions above, along with two others: sequence:adjust-sequence, which is similar to the adjust-array function, but generalized to sequences; and sequence:make-sequence-like, which creates a sequence of the the same kind ${ }^{7}$ as its first argument, with length given by its second argument.

The rationale for make-sequence-like is to support the subseq and copy-seq operators, and the sequence functions which are defined to return a freshly-consed sequence (such as substitute). Additionally, this operator is easier both to specify and to implement efficiently than one like the make-sequence function, which requires a full understanding of the Common Lisp type system; make-sequence-like can operate as a simple generic function specialized on its sequence argument.

To support the delete and delete-duplicates functions, we provide adjust-sequence, which adjusts various properties, including the length, of a sequence. Users of this protocol are encouraged to document the circumstances under which their methods on this operator will preserve the identity of its sequence argument, and must arrange that if a new sequence is allocated, the original sequence argument must not be modified; however, in general, callers (either explicit or implicit) may not assume that the sequence returned from adjust-sequence is the same sequence as its argument.

Once methods for these generic functions have been implemented for a sequence class, all the regular Common Lisp sequence functionality will work as expected. However, some sequences may not admit implementations of all these operations, or indeed might offer means to implement certain operations more efficiently, so in the following sections we describe the protocol by which the Lisp implementation provides the sequence functionality.

\subsection{Iteration}

It is common to iterate over sequences, both in the conception of many of the Common Lisp sequence functions, and in user-defined operations. In this section, we describe the protocol and interface for iterating over sequence contents, both built-in and user-defined. The protocol discussed here has a set of default methods specialized on the sequence class, so that any sequence class for which methods on the protocol functions of section 2.1 have been implemented will obey the protocol. However, these default methods cannot take into account the characteristics of any particular sequence class, and so for efficiency users may wish to override them for their own classes.

The essential concept is the use of an iterator object to represent the current state of an iteration. It is not necessary for this iterator object to have a distinguished class, as the sequence over which it is iterating is present in all function calls in this protocol (and therefore its class can be used for specialization of methods); indeed, the conceptual object is

${ }^{7}$ Neither 'type' nor 'class' is quite right here, as cons and null are distinct types and classes, while both being subtypes of the sequence type list represented in this protocol by three objects and six functions. This iteration protocol is close to the one available in Dylan (Shalit, 1996).

The make-sequence-iterator operator constructs one of these iterator objects: after the required sequence argument, it accepts keyword :start, :end and :from-end arguments, and returns nine values. The first three of those values are an iterator state, a limit and the from-end argument; the remaining six are functions which, respectively, return a state one step ahead, if possible; test the state against the limit for termination; retrieve the element at the current iteration state from the sequence; set the element at the current iteration state to a new value; return the index corresponding to the current iteration state; and return a distinct iteration state representing a copy of the current one.

The default method on make-sequence-iterator is intended for convenience: for most uses, it is unnecessary to construct the nine return values; instead, the default method (specialized to sequence) on make-sequence-iterator generates the first three of the return values by calling makesimple-sequence-iterator, and returns in addition six protocol generic functions: \#'iterator-step (which advances the iteration state); \#'iterator-endp, testing an iteration for termination; the \#'iterator-element reader and the \#' (setf iterator-element) writer; \#'iteratorindex, returning the sequence index corresponding to the iterator state; and finally \#' iterator-copy, returning a copy of the iteration state. These functions have methods specialized to list and vector to provide iterators for the built-in sequence classes.

While implementors of sequence classes may choose to use this CLOS-based iterator protocol (at the potential loss of efficiency through generic function dispatch at each step), users of the iteration protocol (who define functions which perform iterations over sequences) may not assume that the sequence class implementor has done so, and so must call make-sequence-iterator or the operators discussed below.

A small dose of syntactic sugar around make-sequenceiterator is provided by with-sequence-iterator, which binds as if by multiple-value-bind the variables in its first argument to the result of applying make-sequenceiterator to its second argument, and then executes the body. A slightly simpler macro to use is with-sequenceiterator-functions, which binds the six names in its first argument to six local functions (which have dynamic extent and close over the return values from make-sequenceiterator) which perform the various iterator manipulations.

For programmer convenience, we also provide a dosequence macro, behaving as dolist (but for arbitrary sequences), and an extension for loop using the loop keywords element and elements, in a similar fashion to the for-as-package loop path (Pitman and Chapman, 1994, Section 6.1.2.1.7).

It is intended that the iteration protocol described here will be compatible with an iteration protocol for general collections (including hash tables, trees and other similar data structures); at the minimum, any such protocol should be 


\begin{tabular}{|l|l|l|}
\hline some & every & notany \\
\hline notevery & map & map-into \\
\hline concatenate & $\begin{array}{ll}\text { merge } \\
\text { maerce }\end{array}$ & make-sequence \\
\hline
\end{tabular}

Table 3: Common Lisp functions applicable or otherwise relevant to sequences which are not specified as extensible in this protocol; however, the implementation's versions are specified to be applicable to arbitrary sequence types, and to produce the expected result.

able to specify its behaviour for sequences in terms of the operators described here.

\subsection{Sequence Functions}

For most of the functions in the sequences chapter of the standard, there is an analogous generic function with the same argument list which can be specialized for user-defined sequence classes. The complete list of generic functions specified to be extensible, and their corresponding Common Lisp function, is given in table 2 Implementations of this proposal may choose to make the Common Lisp function eql to the extensible generic function, or even make the symbols themselves eql to each other; however, users may not assume this.

\subsection{Other Affected Functions}

The Common Lisp functions in table 3 are not extensible in this proposal. We discuss the interaction of this proposal with the behaviour of those functions below; in summary, where ANSI CL specifies that they accept sequence arguments, the implementation must accept any user-defined sequence, and where ANSI CL specifies that they accept a sequence type-specifer, they must accept a user-defined sequence name or class object (see also Rhodes (2006) for a discussion of the implications of this with respect to the standard behaviour).

Six functions in the SEQUENCES chapter of the ANSI CL standard do not have the structure for user-extensibility in the same straightforward way as the functions discussed in section 2.3 they are concatenate map merge make-sequence, coerce and map-into.

Taking map-into first, we simply specify that an implementation of user-extensible sequences must implement the map-into function such that target sequences and source sequences of arbitrary subtype of sequence are supported given a complete implementation of the sequence and iteration protocol of sections 2.1 and 2.2 An implementation is not prohibited from providing a means of customizing the behaviour of map-into, but neither is it required to.

A means of extending the behaviour of concatenate, map, merge make-sequence and coerce is likewise left unspecified, though again an implementation of this document must provide for the functionality of these functions to be available for objects of arbitrary sequence type and type specifiers naming a concrete subtype of sequence for details of how this interacts with the ANSI CL specified behaviour of these functions, see Rhodes (2006). It is likely that any documented fashion of extending these five functions will use the same mechanism for all of them.

As for functions which are not in the SEQUENCES chapter of the ANSI CL specification, the intent is that where a particular behaviour is specified for a sequence argument, that behaviour should be implemented for the user-extensible sequences. For instance, the short-circuiting quantifiers some every, notevery and notany should accept sequence arguments of arbitrary class; make-array/s : initial-contents argument (and the \#a array reader) should accept a sequence of sequences, as specified, including user-extended sequences. Note that we do not specify a different behaviour of the equalp function from the standard-mandated one (of using eq on objects of type standard-object): although equalp is specified to descend arrays and conses, it does not do so in a sequence-like way, and in particular a list and a vector with the same content are not considered to be equalp. Equality over sequence contents can be computed with mismatch

Particular attention must be paid to the read-sequence and write-sequence functions. Although Gray (1989) does not suggest that these functions be extensible in the manner of the other stream functions, it is likely that this is because read-sequence and write-sequence were added to the language after the extensible streams proposal was made, and indeed Common Lisp implementations have provided stream-read-sequence and stream-write-sequence generic functions for user customizeability. Lisps providing both an implementation of this proposal and extensible streams based on Gray (1989) should document the effect of calling read-sequence and write-sequence with arguments being instances of non-standardized classes. The simplestreams (Franz Inc., 2006. Chapter 83) extensible analogue is documented to signal an error for all sequence types other than strings and octet vectors; if this restriction is relaxed, there are similar interactions as with Gray streams over which extensibility (sequence or stream) takes precedence.

\section{EXAMPLES}

In this section, we present two examples of uses for the protocols discussed in this paper. Firstly, in section 3.1. we present the implementation of a distinct sequence type along with the method definitions to allow it to interoperate; then, in section 3.2, we demonstrate the definition of a mixin class and methods specialized on it to provide additional functionality to generic sequences implemented as in this paper.

\subsection{Queue}

As an example of a non-standard sequence, consider implementing a queue data structure, which supports the operations enqueue and dequeue. Figure 2 shows one way in which such a queue could be implemented, using a list as the storage for the data, and keeping a reference to the cons cell at the back of the queue. Purely for interest, we also implement a variant of the queue which is also funcallable, and arrange so that calling the funcallable queue with no arguments performs dequeueing, while with one argument it enqueues that argument.

An implementation of the sequence and iteration protocol 




Figure 2: Basic definitions of a queue data structure, including a funcallable variant. The layout of the code in this and subsequent figures is not idiomatic for reasons of space.

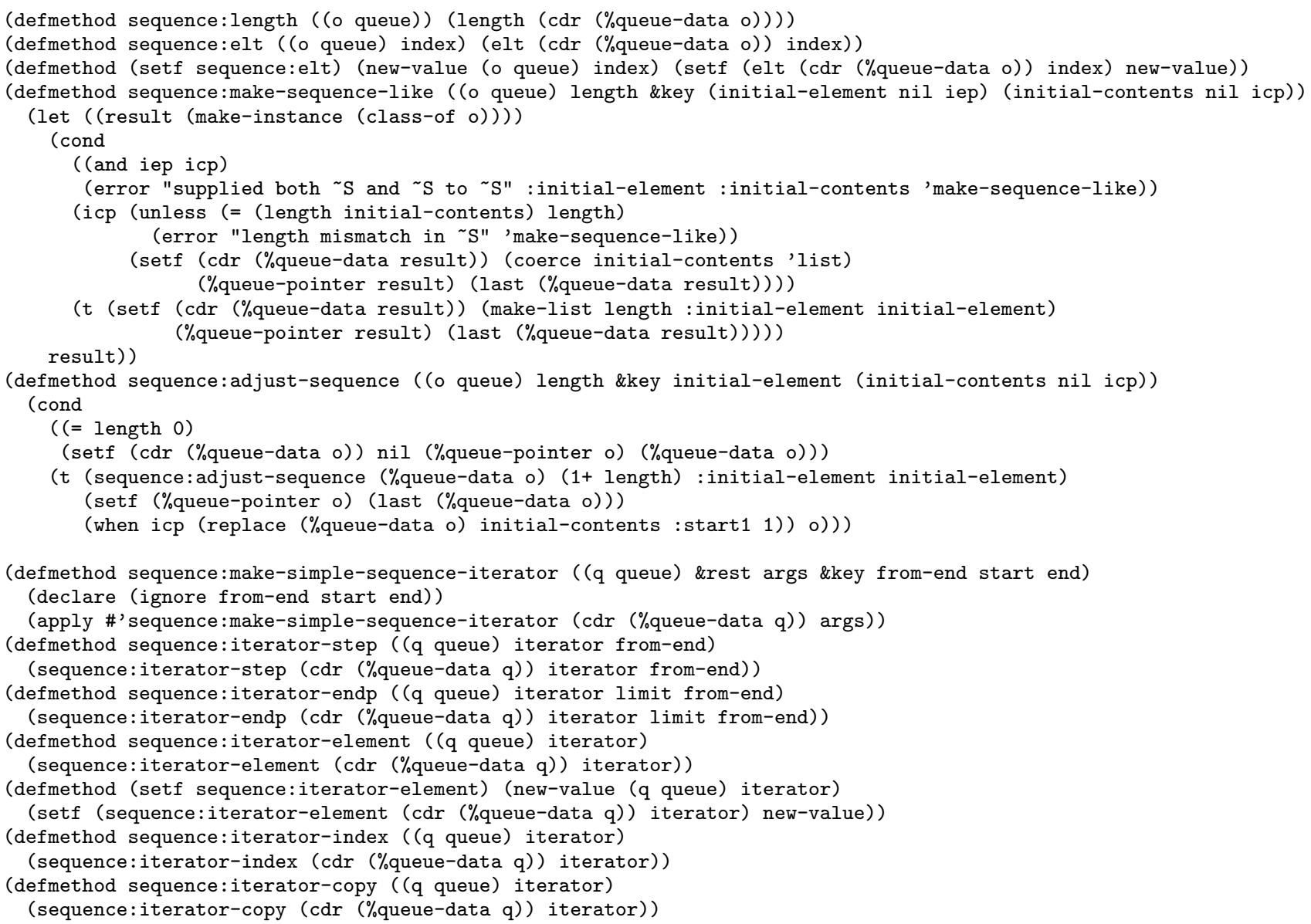

Figure 3: Implementation of the sequence protocol for the queues of figure 2, The first five method definitions are sufficient to allow all sequence functionality to run correctly; the methods on the iterator functions reduce the complexity for our simple queue implementation from $O\left(N^{2}\right)$ to $O(N)$ for most operations. 


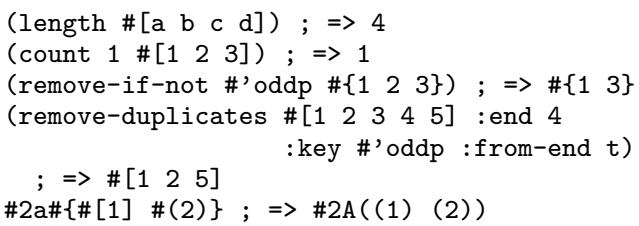

Figure 4: Examples of using the queues as sequences. For the \#[ and \#\{ reader macros and queue print functions, see figure 7 in the appendix.

for a queue implemented in this fashion is shown in figure 3 . The first five method definitions are required under this proposal, for implementing the primitive sequence operations. The default method on make-sequence-iterator would suffice to make all Common Lisp sequence functionality work; however, an index-based iteration scheme as provided by that default method would suffer from poor performance for our implementation of queues based on lists, so we define methods for the iterator functions of section 2.2 , calling the system-provided methods for lists (assumed to be efficient at least for forward iteration).

Given the code in figure 3, queues can be used wherever Common Lisp specifies that a sequence is acceptable. For instance, one can ask for the position of an element in the queue using position, with all the keywords (:test, :key, :start etc.) that the Common Lisp function accepts; some examples are given in figure 4. Furthermore, this queue implementation will potentially interoperate with bodies of code written for generic sequences, as long as there are no uses of the formal interpretation of the requirements of make-sequence and friends.

\subsection{Undoable mixin}

To illustrate possible uses of the sequence protocol discussed in this document, we present in figure 5 a mixin for implementing some undo functionality for a sequence. The undoable-mixin class contains a record slot, which records enough information to reconstruct the state of the sequence before an operation; if the user calls the undo function on a sequence with this class mixed in, the previous state of the sequence will be reconstructed using undo-using-record methods. A more sophisticated version of this might be useful as a component of an editor buffer implementation, for example.

For (setf elt), we record the index and the value at that index before performing the mutation; clearly, this permits reconstructing the previous state of the sequence. For operations such as fill and nreverse, we could simply make do with this (and an analogous change for the setter function of the iterator protocol; see figure 8 in the appendix for that detail), but this would have the consequence that a single logical operation such as fill would require multiple calls to undo to undo the state.

Instead, therefore, for fill, we record the contents between the bounding index designators, while for nreverse we need record nothing, as it is a reversible operation 8 An alter-

\footnotetext{
${ }^{8} \mathrm{We}$ assume for expository purposes that the destructive
}

native strategy for grouping multiple primitive operations, used in the method for recording calls to nsubstitute and delete, is shown in figure 8 in the appendix.

\section{IMPLEMENTATION DETAILS}

We have implemented the above proposal in Steel Bank Common Lisp (Newman et al., 2000); the overall strategy was to modify the implementations of the standard Common Lisp sequence functions to call into their sequence package analogues instead of signalling an error, if their sequence arguments were neither lists nor vectors. This way of implementating this proposal was motivated by changing the run-time performance characterstics of the system as little as possible for existing code.

The first aspect of SBCL itself which needed to be modified was the type system: the system needed to be informed that sequence was no longer simply an alias in that implementation of (or list vector), but was an unsealed (that is, subclassable) class with its own identity in the type system; since the SBCL compiler makes heavy use of type inference, both when compiling user code and when cross-compiling itself, it was also important for the compiler's version of subtypep to know that the types (and sequence vector) and (and sequence list) are equivalent to vector and list respectively, irrespective of the extensibility of sequence

Additionally, the declared return type of various sequence functions such as copy-seq needed to be altered: SBCL has the type consed-sequence, which was previously aliased to (or list (simple-array * (*))), expressing that (in SBCL) freshly consed vectors do not have fill-pointers, are not displaced and are not adjustable. In an implementation supporting extensible sequences, this type alias needs to be changed to (or (simple-array $*(*)$ ) (and sequence (not vector) )) to correctly represent the implementation's behaviour

After these modifications to the type system, the implementation of the remainder of this proposal involved two distinct parts: modifying SBCL itself to insert calls to the sequence generic functions (say, sequence: length) from the standard functions (length), and implementing those generic functions in a userspace library. Incremental development of the SBCL internals was eased by the use of a seq-dispatch macro, taking a sequence argument and expanding into either two or three cases: one for when the argument is a list, one when it is a vector, and (optionally) one for neither: the third case is only executed when a generic sequence is encountered.

The various compiler transformations and optimizations of sequence functions (such as find, position, map, coerce) were largely unaffected, as either the various checks on their applicability were already restrictive enough, or else the optimizations performed were generic to all sequences. The implementation of find and position (and -if and -ifnot relatives) needed a small amount of alteration to allow

sequence functions such as nreverse act in-place on the userextended sequences in which this class will be mixed.

${ }^{9} \mathrm{SBCL}$ has a sophisticated understanding of the Common Lisp type system, so such complex types do not cause difficulty in type inference. 


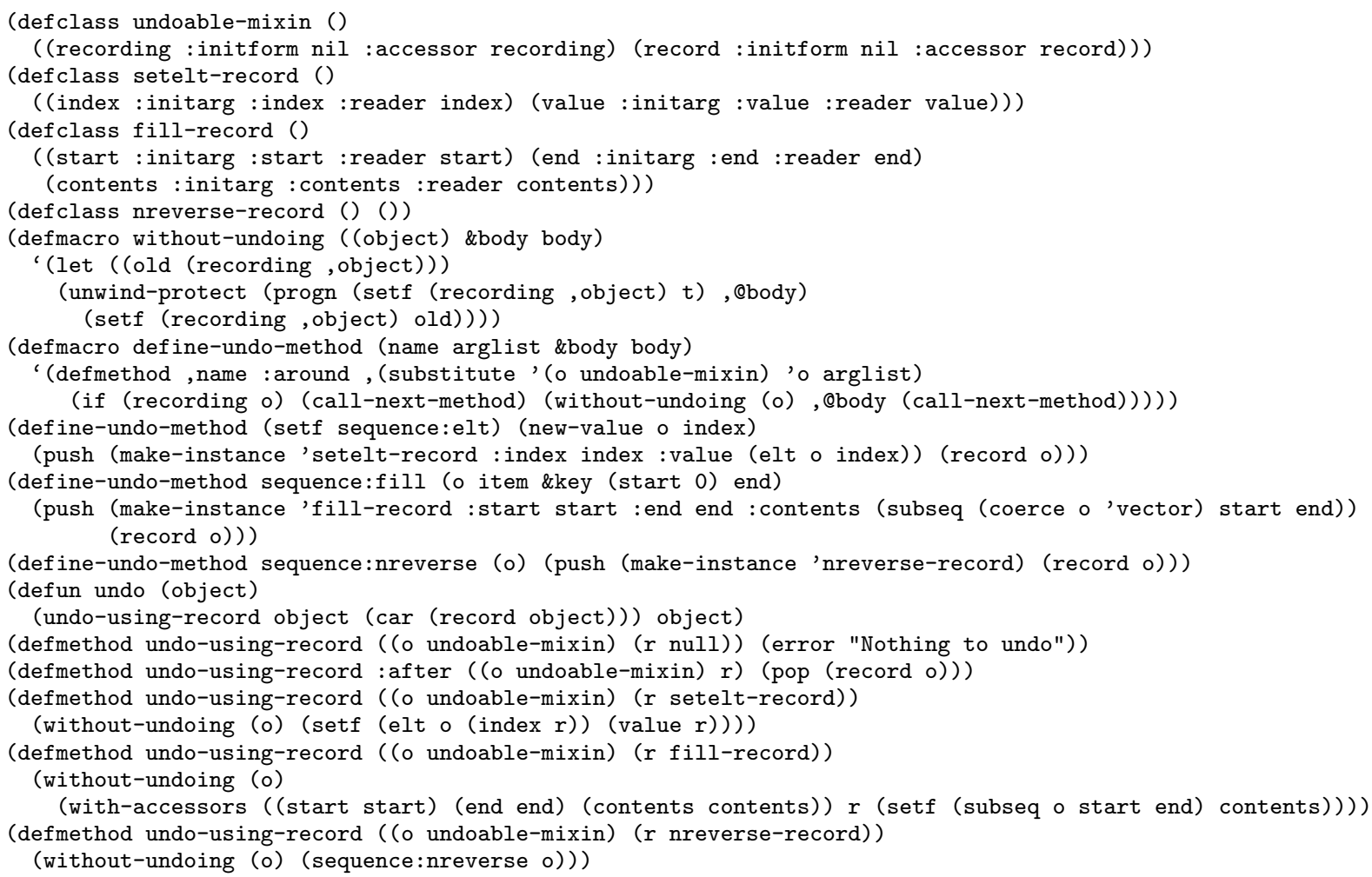

Figure 5: Illustration of a simple implementation of undo for sequences: an undoable-mixin class which can be mixed in to a sequence class, providing undo functionality (illustrated here for (setf elt), fill and nreverse).

the protocol for sequence: find and sequence:position described in section A.3 to be implemented, while the implementation of map was improved in the process of development, being rewritten to use dynamic-extent support, resolving a long-standing issue in the system.

Support for defining subclasses of sequence from the customized PCL (Bobrow and Kiczales, 1988) which SBCL incorporates to implement CLOS was as simple as modifying the system method for the MetaObject Protocol function mop:validate-superclass to allow sequence as a direct superclass of classes with metaclasses standard-class and mop: funcallable-standard-class. In addition, to support the operations involving sequence type specifiers (such as map and merge), we used make-sequence-like acting on the mop:class-prototype of the class named by the type specifier, if that class was a subclass of sequence

SBCL's 1oop facility is based on the MIT implementation, so it was straightforwardly extended using the add-loop-path operator as shown in figure 6 .

In the implementation of this proposal for SBCL, there is no run-time performance impact for bodies of code which does not use the facilities provided by user-extensible sequences (see http://sbcl.boinkor.net/bench/ for benchmark data: the relevant changeset is labelled 1.0.0.22). This is understandable, as all that has been done to the runtime code in this implementation is to convert cases which used to signal errors (usually the type-error for the datum not being a sequence) into calls to the analogous function of the sequence package. Changes in compile-time performance are potentially present but unmeasurable: the only likely contributor is the additional work in computing type relationships between sequence and other types. There is an increase of approximately $1 \%$ of the heap size of a fresh SBCL instance, because of the extra code used in implementing this proposal; however, this extra space is in a tenured generation and behind a write barrier, and therefore does not significantly increase garbage-collection times.

\section{CONCLUSIONS}

We have described an extension to Common Lisp to allow users to define their own sequence classes in an interoperable manner, and have described the changes necessary to implement this extension in a contemporary implementation. Additionally, we have attempted to justify the need for this extension in terms of expressivity and parsimony, and given simple examples of using it.

We leave for future work the related task of defining a protocol for user-defined collections; Common Lisp sequences are a specialized form of collection, finite and ordered. However, preliminary work suggests that such a collection protocol can be defined to interoperate with the proposals in this document for sequences.

It is intended that a revised and expanded version of appendix A should be submitted to some suitable forum for standardization of some form; it is likely that feedback gath- 




Figure 6: Extension of loop for a loop path to iterate over the elements of generic sequences.

ered from discussion of this paper will mean that there will be some changes in detail in the revision process, so users should not rely on the description in this paper remaining authoritative.

\section{Acknowledgments}

We thank Nikodemus Siivola, Marcus Pearce, Gábor Melis, Eric Marsden, Peter Housel, Cyrus Harmon, Peter Graves and Pascal Costanza for fruitful and helpful discussions, and acknowledge financial support from EPSRC grant number GR/S84750/01. We also wish to thank the reviewers for their valuable feedback.

\section{References}

Bobrow, D. G. and Kiczales, G. (1988). The Common Lisp Object System Metaobject Kernel: A Status Report. In Lisp and Functional Programming, pages 309-315.

Franz Inc. (2006). Allegro CL version 8.0 documentation. http://tinyurl.com/3cfu66

Gray, D. N. (1989). Issue STREAM-DEFINITION-BY-USER. Failed Issue, X3J13, ANSI. http://tinyurl.com/2d4csd.

Haible, B. (1988). The Abstract Datatype Sequence. Technical report, University of Karlsruhe. http://tinyurl. com/yy3eys

Haible, B. (2006). personal communication.

Kelsey, R., Clinger, W., and Rees, J. (1998). Revised ${ }^{5}$ Report on the Algorithmic Language Scheme. Higher-Order and Symbolic Computation, 11(1).

Kiczales, G., des Rivières, J., and Bobrow, D. G. (1991). The Art of the Metaobject Protocol. MIT Press.

Kiczales, G., Lamping, J., Menhdhekar, A., Maeda, C., Lopes, C., Loingtier, J.-M., and Irwin, J. (1997). AspectOriented Programming. In European Conference on Object-Oriented Programming, pages 220-242.

Miller, S. G. (2004). Collections. SRFI 44, schemers.org. http://srfi.schemers.org/srfi-44/srfi-44.html

Newman, W. H. et al. (2000). SBCL User Manual. http: //www.sbcl.org/manual/
Norvig, P. (2002). Large-Scale Web Services, and the Programming Languages that Build Them. In International Lisp Conference Proceedings.

Pestov, S. (2006). Factor documentation. http:// factorcode.org/responder/help/

Pitman, K. and Chapman, K., editors (1994). Information Technology - Programming Language - Common Lisp. Number 226-1994 in INCITS. ANSI.

Pitman, K. M. (1991). Issue CONCATENATE-SEQUENCE. Issue 73, X3J13, ANSI. http://tinyurl.com/2zt4d6

Rhodes, C. (2006). Revisiting CONCATENATE-SEQUENCE. Document 3, Common Lisp Document Repository. http: //cdr.eurolisp.org/document/3

Rhodes, C., Strandh, R., and Mastenbrook, B. (2005). Syntax Analysis in the Climacs Text Editor. In International Lisp Conference Proceedings.

Shalit, A. (1996). The Dylan Reference Manual. AddisonWesley, Redwood City, CA, USA. http://www . opendylan.org/books/drm/Title

Steele, Jr, G. L. (1990). Common Lisp: The Language. Digital Press, second edition.

Strandh, R., Villeneuve, M., and Moore, T. (2004). Flexichain: An editable sequence and its gap-buffer implementation. In European Lisp and Scheme Workshop. http://tinyurl.com/yhqwp3

Yee, K.-P. and van Rossum, G. (2001). Iterators. PEP 234, Python Software Foundation. http://www.python.org/ dev/peps/pep-0234/

\section{APPENDIX \\ A. SPECIFICATION}

In the sections below, all of the generic functions and macros being specified are named by symbols external in the sequence package. In cases where the generic function being defined corresponds to a standardized Common Lisp function, it is not specified whether the corresponding Common Lisp function is distinct from the specified 


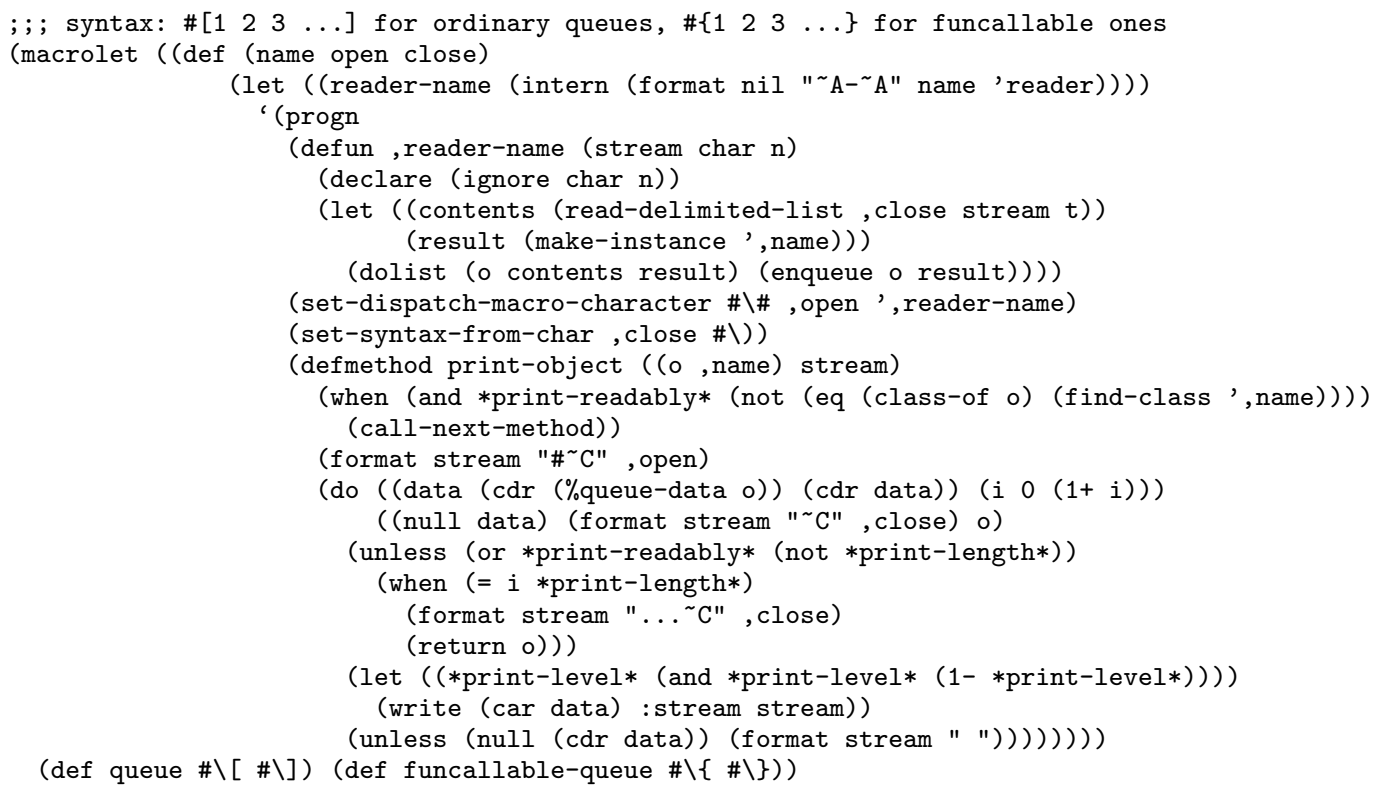

Figure 7: Implementation of reader macros and print functions for queues and funcallable queues. Note the support for correct behaviour in the presence of non-nil values of printer control variables.

generic functions, nor indeed whether the symbol in the common-lisp package is distinct from that in the sequence package; that is, \#'length might or might not be eql to \#'sequence: length, and length might or might not be eql to sequence:length. The sequence package may have additional, implementation-specific names; sequence need not be the package-name of the package.

It is not specified whether the methods specified on list and vector are in fact called when the Common Lisp function corresponding to the generic function is called on such data. It is not specified whether the methods specified on sequence are called when the Common Lisp function corresponding to the generic function is called on data of type vector or list.

In implementations which support the Metaobject Protocol as defined in Kiczales et al. (1991), suitable methods on mop: validate-superclass should be provided such that no error is signalled for user-defined sequence classes of metaclass standard-class such implementations are additionally encouraged to allow mop:funcallable-standard-class as a compatible metaclass for user-defined sequence classes.

\section{A.1 Basic sequence operations}

We specify generic functions length, elt and (setf elt) to correspond to the Common Lisp functions with the same name. In each case, there are two primary methods with the sequence argument specialized on list and on vector, providing the standard-defined behaviour for the Common Lisp operator, and a third method with the sequence argument specialized on sequence, which signals an error of type typeerror for compatibility with the standard requirement of the sequence argument to be a proper sequence.

In addition, we specify two new generic functions and corresponding methods (with a similar pattern of behaviour) described below.

Generic Function make-sequence-like

Syntax:

make-sequence-like sequence length \&key initial-element initial-contents

This generic function returns a sequence of the same class as its sequence argument, with the specified length. If neither initial-element nor initial-contents is supplied, the consequences are undefined if any element of the resulting sequence is read before being written. If initial-element is provided, it is used to initialize the resulting sequence; if initial-contents is provided, it must be a sequence of length length, which is used to initialize the resulting sequence. The consequences are undefined if both initial-element and initial-contents are supplied.

Primary Method make-sequence-like ( $l$ list) length \&key initial-element initial-contents

Primary Method make-sequence-like ( $v$ vector) length \&key initial-element initial-contents

No behaviour for these methods is specified beyond that for the generic function.

Primary Method make-sequence-like ( $s$ sequence) length \&key initial-element initial-contents

This method signals an error of type type-error.

Generic Function adjust-sequence

Syntax:

adjust-sequence sequence length \&key initial-element initial-contents 




(define-compound-undo-method sequence:nsubstitute (new old o \&key from-end start end test test-not key count)) (define-compound-undo-method sequence:nsubstitute-if (new old o \&key from-end start end key count))

(define-compound-undo-method sequence:nsubstitute-if-not (new old o \&key from-end start end key count))

(defclass adjust-sequence-record ()

((previous : initarg :previous :reader previous) (discarded :initarg :discarded :reader discarded)))

(define-undo-method sequence:adjust-sequence (o length \&rest args)

(push (make-instance 'adjust-sequence-record :previous (length o) (record o)))

(defmethod undo-using-record ( (o undoable-mixin) ( $r$ adjust-sequence-record))

(sequence:adjust-sequence o (previous $r$ ))

(when (discarded $r)$ (setf (subseq o (- (length o) (length (discarded $r)))$ ) (discarded $r)))$ )

(define-compound-undo-method sequence:delete (item o \&key from-end (start 0) end test test-not key count)) (define-compound-undo-method sequence:delete-if (item o \&key from-end (start 0 ) end key count))

(define-compound-undo-method sequence:delete-if-not (item o \&key from-end (start 0) end key count))

(define-compound-undo-method sequence:delete-duplicates (o \&key from-end (start 0) end test test-not key))

(define-undo-method sequence:replace (o sequence2 \&key (start1 0 ) end1 (start2 0) end2)

(push (make-instance 'fill-record :start start1 :end end1 : contents (coerce (subseq o start1 end1) 'vector)) $($ record o)) )

(define-undo-method sequence:sort (o predicate \&key key)

(push (make-instance 'fill-record : contents (coerce o'vector) :start 0 : end nil) (record o)))

(define-undo-method sequence:stable-sort (o predicate \&key key)

(push (make-instance 'fill-record :contents (coerce o 'vector) :start 0 : end nil) (record o)))

Figure 8: Implementation details of the undoable mixin. The setter in the iterator protocol is wrapped by the : around method on make-sequence-iterator; a specialized undo record is defined for adjust-sequence; and undo records for nsubstitute and delete are represented as sequences of primitive undo records, treated as an atom for the purposes of running undo. 
This generic function changes the length or elements of sequence. It returns a sequence of length length, with contents as specified by initial-contents if provided, otherwise with the original contents in their original position and initialelement as any additional elements. The return value may, but need not, share structure with or be identical to the sequence argument.

Primary Method adjust-sequence ( $l$ list) length \&key initial-element initial-contents

Primary Method adjust-sequence ( $v$ vector) length \&key initial-element initial-contents

This method functions in a similar manner to adjust-array, though the implementation may choose to preserve the identity of the argument if it has a fill pointer and the length argument is not greater than the size of the vector.

Primary Method adjust-sequence ( $s$ sequence) length \&key initial-element initial-contents

This method signals an error of type type-error

\section{A.2 Iteration}

\author{
Generic Function make-sequence-iterator \\ Syntax: \\ make-sequence-iterator sequence \&key from-end start \\ end
}

This generic function returns nine values: three values corresponding to an iterator state, a limit state and from-end, and six functions with signatures and functionality like the iterator-functions below.

\section{Primary Method make-sequence-iterator ( $s$ sequence) \&key from-end (start 0) end}

This method returns the three values returned by calling make-simple-sequence-iterator, along with the iterator functions \#'iterator-step, \#'iterator-endp, \#'iteratorelement, \#'(setf iterator-element), \#'iterator-index and \#'iterator-copy defined below.

For the seven following generic functions, we describe both the generic function and also in more detail the return values of the primary method, specialized on sequence The primary methods described here are suitable for iteration across data structures with efficient random access, such as vectors, and less suitable for iteration across some other data structures, such as lists. We expect implementations of this protocol to provide additional methods to perform more efficient iteration on lists than is possible with the primary method described; however, we do not impose additional requirements on the behaviour of such methods.

\section{Generic Function make-simple-sequence-iterator Syntax: \\ make-simple-sequence-iterator sequence \&key from-end start end}

This generic function returns three values: an iterator object, a limit state and from-end. These values, along with sequence, are to be used for calling to the iterator generic functions, below; the consequences are unspecified if objects not returned by a call to make-simple-sequence-iterator are passed as arguments to the iterator functions. The primary method returns the three values start, end (or the length of sequence if end is nil) and nil, if from-end is false; if from-end is true, it returns the three values (1- (or end (length sequence))), (1- start), and from-end.

\section{Generic Function iterator-step \\ Syntax: \\ iterator-step sequence iterator from-end}

This generic function returns a new iterator state representing the advancement of the iteration across sequence in the direction indicated by from-end. The primary method returns (1- iterator) if from-end is true; otherwise, it returns $(1+$ iterator $)$.

\section{Generic Function iterator-endp \\ Syntax: \\ iterator-endp sequence iterator limit from-end}

This generic function tests the iterator for having reached its end state for iteration across sequence indicated in the direction indicated by from-end. The primary method returns the value of (= iterator limit) .

\section{Generic Function iterator-element \\ Syntax: \\ iterator-element sequence iterator}

This generic function returns the element of sequence at the point of iteration indicated by iterator. The primary method returns the value of (elt $s$ iterator).

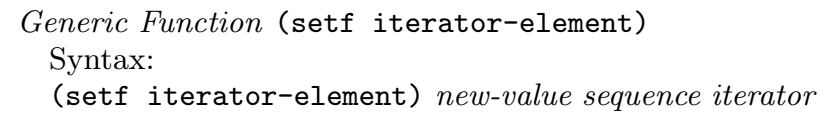

This generic function sets the element of sequence at the point of iteration indicated by iterator to new-value. The primary method returns the value of ( $\operatorname{setf}$ (elt $s$ iterator) new-value).

\section{Generic Function iterator-index Syntax: \\ iterator-index sequence iterator}

This generic function returns the index of the point indicated by iterator in sequence. The primary method returns iterator.

\section{Generic Function iterator-copy Syntax: \\ iterator-copy sequence iterator}

This generic function returns an iterator state identifying the same point in an iteration as iterator, such that changes to one do not affect the other. The primary method returns iterator.

\footnotetext{
Macro with-sequence-iterator (\&optional state limit from-end step endp elt setelt index copy) (sequence \&key from-end (start 0) end) \&body body
} 
This macro binds the names in its first argument list as if by multiple-value-bind to the values returned by makesequence-iterator applied to its second argument list, and then executes body.

\section{Macro dosequence (var sequence-form \&optional result-form) \&body body}

This macro iterates over a sequence, in a similar fashion to dolist iterating over a list. The body is like a tagbody, consisting of a series of tags and statements.

dosequence evaluates sequence-form, which should produce a sequence. It then executes the body once for each element in the sequence, in the order in which the tags and statements occur, with var bound to the element. Then result-form is evaluated with var bound to nil

An implicit block named nil surrounds the entire dosequence form. return may be used to terminate the loop immediately without performing any further iterations, returning zero or more values.

The scope of the binding of var does not include the sequence-form, but the result-form is included.

It is implementation-dependent whether dosequence establishes a new binding of var on each iteration or whether it establishes a binding for var once at the beginning and then assigns it on any subsequent iterations.

\section{Loop Path for-as-sequence}

This allows using an iteration variable, as with other loop for-as-clauses, as if the following clause were added to the loop grammar (Pitman and Chapman, 1994, Macro 1oop):

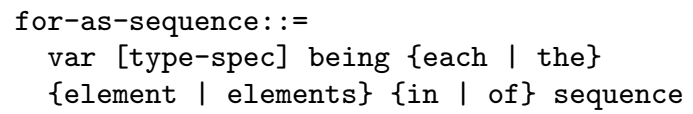

and as if the for-as-subclause clause permitted this foras-sequence clause along with the standardized clauses. As with other iteration control clauses, the variable argument may be a destructuring list. The effect of this clause is to iterate through the contents of a sequence, starting from the zeroth element and terminating at the end of the sequence ${ }^{10}$

\section{A.3 Sequence Function Specifications}

Except as specified here, it is implementation-dependent whether methods on these generic functions call other such generic functions or not. For each of these generic functions there is a method, called the "default method" below, where all sequence parameters are specialized on sequence implementing the default behaviour given an implementation of the iteration and basic sequence protocol in the sections above. The user of this protocol is permitted to extend or override these generic functions, but is not permitted to specialize any non-sequence argument.

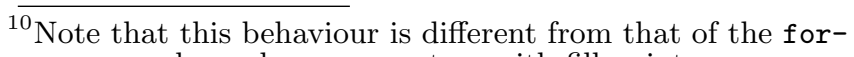
as-across loop clause on vectors with fill pointers.
It is unspecified whether the generic functions specified below are called when the corresponding Common Lisp function has only list and vector arguments for sequences; however, the implementation is required to provide methods for these generic functions implementing similar behaviour, so that the user may call these generic functions on list and vector sequences.

For each Common Lisp function in table 2 we specify a generic function of the same name in the sequence package with the same argument list, each with a default method where all sequence arguments are specialized on sequence Given that the sequence argument to these functions is named sequence, we enumerate the specified calling relationships between these functions as follows:

- the default method on copy-seq calls subseq with parameters sequence and 0 ;

- the default method on substitute calls copy-seq on sequence, and then calls nsubstitute on its arguments with the provided sequence replaced by the copy. This pattern is repeated for the -if and -if-not variants of substitute, and for remove, remove-if, remove-ifnot and remove-duplicates calling the corresponding delete-function.

- the default method on sort behaves as if it constructs a vector with the same elements as sequence, calls sort on that vector, then replaces the elements of sequence with the elements of the sorted vector.

The rationale behind specifying these calling relationships is to make clear the work needed by a sequence class implementor to install specialized implementations of these functions for particular sequence classes. 